\title{
Accelerated Learning and Assessment in Engineering Mechanics: Designing an Interactive Tool to Support Students' Learning
}

\section{Dr. Nicole P. Pitterson, Virginia Polytechnic Institute \& State University}

Nicole is an assistant professor in the Department of Engineering Education at Virginia Tech. Prior to joining VT, Dr. Pitterson was a postdoctoral scholar at Oregon State University. She holds a PhD in Engineering Education from Purdue University and other degrees in Manufacturing Engineering from Western Illinois University and a B.Sc. in Electrical and Electronic Engineering from the University of Technology, Jamaica. Her research interests are exploring students' disciplinary identity through engagement with knowledge, curriculum design, assessment and evaluation and teaching for conceptual understanding.

\section{Dr. Jacob R Grohs, Virginia Polytechnic Institute and State University}

Jacob Grohs is an Assistant Professor in Engineering Education at Virginia Tech with Affiliate Faculty status in Biomedical Engineering and Mechanics and the Learning Sciences and Technologies at Virginia Tech. He holds degrees in Engineering Mechanics (BS, MS) and in Educational Psychology (MAEd, $\mathrm{PhD})$.

\section{Prof. David A. Dillard, Virginia Tech}

David Dillard is the Adhesive and Sealant Science Professor in the Biomedical Engineering and Mechanics Department at Virginia Tech. He has worked extensively in the field of adhesive bonding, having experience in structural adhesives for aerospace, automotive, and infrastructure applications; adhesives and coatings for microelectronic applications; pressure sensitive adhesives; elastomeric adhesives and sealants; and polymeric membranes. He has authored or co-authored over 190 refereed publications and regularly teaches courses in adhesion science, polymer viscoelasticity, and sustainable energy solutions. With nearly 40 years of experience as an educator, he is interested in new ways to leverage technology to have a positive impact on student learning and assessment.

\section{Dr. Sneha Patel Davison, Virginia Polytechnic Institute and State University \\ Mr. Todd P. Shuba, Virginia Tech}

Todd P. Shuba is a New Horizons Graduate Scholar in the College of Engineering and a Graduate Research and Teaching Assistant in the Department of Engineering Education at the Virginia Polytechnic Institute and State University. His research interests include transfer of learning, collaborative learning, and student motivation and engagement. He holds a Bachelor of Science in Interdisciplinary Engineering with a concentration in Environmental and Ecological Engineering and a minor in Mechanical Engineering, as well as a Master of Science in Education with a concentration in Educational Psychology and Research Methodology, from Purdue University-West Lafayette.

\section{Prof. Cliff Shaffer, Virginia Polytechnic Institute and State University}

Dr. Shaffer received his PhD in Computer Science from University of Maryland, College Park in 1986. He is currently Professor of Computer Science at Virginia Tech, where he has been since 1987. He directs the AlgoViz and OpenDSA projects, whose goals respectively are to support the use of algorithm visualization in the classroom, and the development of a complete online collection of interactive tutorials for data structures and algorithms courses. His research interests are in Computational Biology and Bioinformatics, Problem Solving Environments, Digital Education, Algorithm Visualization, Hierarchical Data Structures, Algorithm Design and Analysis, and Data Structures.

Mr. ARINJOY BASAK, Department of Computer Science, Virginia Tech Mr. Jianqiang Zhang, Virginia Tech 


\title{
Accelerated Learning and Assessment in Engineering Mechanics: Designing an Interactive Tool to Support Students' Learning
}

\begin{abstract}
Repeated deliberate practice in problem-solving practices is known to be beneficial in increasing students' overall understanding of targeted concepts. Additionally, when students receive frequent formative feedback, they are able to identify problematic areas of their reasoning and can correct their underlying cognitive frames of reference. However, many undergraduate engineering courses are not designed to provide students with repeated practice and targeted feedback by use of educational interventions. This project was designed to: 1) iteratively develop the innovative problem delivery and assessment system and evaluate its effectiveness in meeting specific learning and assessment goals in engineering mechanics, 2) systematically study how this technology-rich problem-solving interface can enhance the learning, teaching, and assessment of complex knowledge, and 3) critically evaluate opportunities and barriers to scaling and transferring the innovation across educational contexts. By focusing on the development of strong analytical problem-solving skills characterized by rich conceptual knowledge, this project directly responds to demands from both industry and the federal government for colleges and universities to develop complex problem solvers for the workforce.
\end{abstract}

Overall, this project aims to assist engineering faculty and students through the development of an open-access problem-solving interface that will accelerate learning and enhance assessment, which along with a growing body of practice exercises, could be widely adopted in engineering mechanics education. The project seeks to develop an interactive online system for solving problems in introductory engineering mechanics courses for an undergraduate degree program. Our system aims to provide a feedback-based, exploratory environment for students to work on different problem instances and variations to explore key concepts of mathematical problem solving through developing equations for a target solution. This poster will present the current implementation of our system and discuss the different features for problem solving, feedback, and tracking of student activity and progress.

\section{Background}

The Engineer of 2020 report claims that the engineers of tomorrow must exhibit "practical ingenuity" characterized by an ability to adaptively reason through complex problems, in part drawing on "strong analytical skills" that rely on understanding of fundamental principles of science and mathematics [1] Preparing thinkers and innovators capable of addressing complex problems has long been an espoused focus of engineering education. Recent studies show that problem-solving ability is both an increasingly intentional aspect of engineering curriculum and a fundamental competency demanded by employers [2]. Engineering textbooks, syllabi, and tests provide evidence of these findings. Such course materials are dominated by problem exercises for good reason. Problems offer opportunities for engagement, provide practice and feedback to guide the learning process, and are framed around real-world applications for engineering knowledge. Unfortunately, producing solutions is time-consuming for students, and these solutions are often difficult to assess in other than a right or wrong comparison to answers "in the back of the book". These result in attempting a rather limited number of problems for both practice and assessment, so students do not get as much practice and feedback as they often wish. 
Surprisingly, in spite of tremendous advances in calculators, the time required to work many typical homework or test problems is only slightly reduced from practices involving slide rules that were widely used until 45 years ago, suggesting both the need and opportunity for accelerated problem-solving through appropriate computer interfaces to build skills and confidence solving more and different problems. Despite the prevalence of problem exercises in the learning and assessment of engineering knowledge, recent research suggests a troubling mismatch between what is taught, what is learned, and what is assessed.

\section{The Innovation}

This project aims to develop an open-access, online adaptive problem-solving environment that can enable and foster accelerated learning, offer opportunities to improve classroom efficiency and effectiveness, and enhance assessment accuracy and effectiveness in engineering. Additionally, this environment features a growing body of practice exercises that can be widely adopted. Our materials will be open source, and the novelty of the interface for mechanics problems and our study of their impact on the curricula will contribute to scholarship on learning in technology-rich environments. Our focus is on undergraduate mechanics courses because these form an essential foundation for many engineering disciplines, are primarily taught and assessed through a large portfolio of problem exercises, and have been documented as significant barriers for student persistence and success in engineering [3], [4]. These are often positioned as the last foundational courses before students enroll in specific disciplines (e.g., civil, mechanical, or industrial systems engineering) and conceptually demand that students synthesize knowledge across many first-year engineering and mathematics courses. Class sizes are becoming so large that assessment must often be mechanized. As a result, traditional methods mean limits on the ability of teachers to give targeted, timely feedback [5]. Thus, these courses offer a prime opportunity for us to implement and study the role of our proposed innovation, and the degree to which our problem-based exercises can achieve goals both specific to the undergraduate engineering mechanics context and also be readily scalable beyond.

This project is guided by the following goals:

Goal 1: Iteratively develop the innovative problem delivery and assessment system, and evaluate its effectiveness in meeting specific learning and assessment goals in engineering mechanics; Goal 2: Systematically study how this technology-rich problem-solving interface can enhance the learning, teaching, and assessment of complex knowledge;

Goal 3: Critically evaluate opportunities and barriers to scaling and transferring the innovation across educational contexts;

\section{Current and Future Steps}

Currently, we are in the process of creating a workable prototype. We contend that mechanics students will learn new concepts more rapidly if they can get more relevant practice (with targeted feedback) on a platform designed to reduce the time spent performing calculations by hand. Since students tend to perform mathematical calculations as a ritual without completely understanding the interaction of variables throughout the process, the learning framework being developed can be used to deliver interactive, automatically assessed problems for mechanics and is extendable to broader STEM education. Building on insights from existing open-source work

\section{Page 2 of 4}


in other domains with different goals, we built an online system in which students working exercises are presented with a palette of equations appropriate for the Deformable Bodies course. After selecting relevant equations from the palettes, students identify what terms are used in each equation from highlighted dimensions, material properties, and other parameters given in the problem statement or problem figure. The student is also able to generate their own equations from relevant algebraic and trigonometric functions. Changes in units can be specified. The student is then able to identify to the system the unknown(s) being solved for. By tracking the number of equations involved and the number of variables, the software presents the student with the option to allow the program to solve for the answer(s) once the number of equations and unknown variables permit a solution. The order of equations entered, and variables replaced does not affect the final solution, hence the approach can flexibly accommodate a range of student approaches to problem solving. Through this easily learned click-and-drag format, students can quickly assemble the necessary equations and demonstrate their understanding by associating problem statement quantities with equation variables, obtaining uniquely meaningful feedback on their progress and on the final answer. The figure 1 and 2 below demonstrates the prototype.

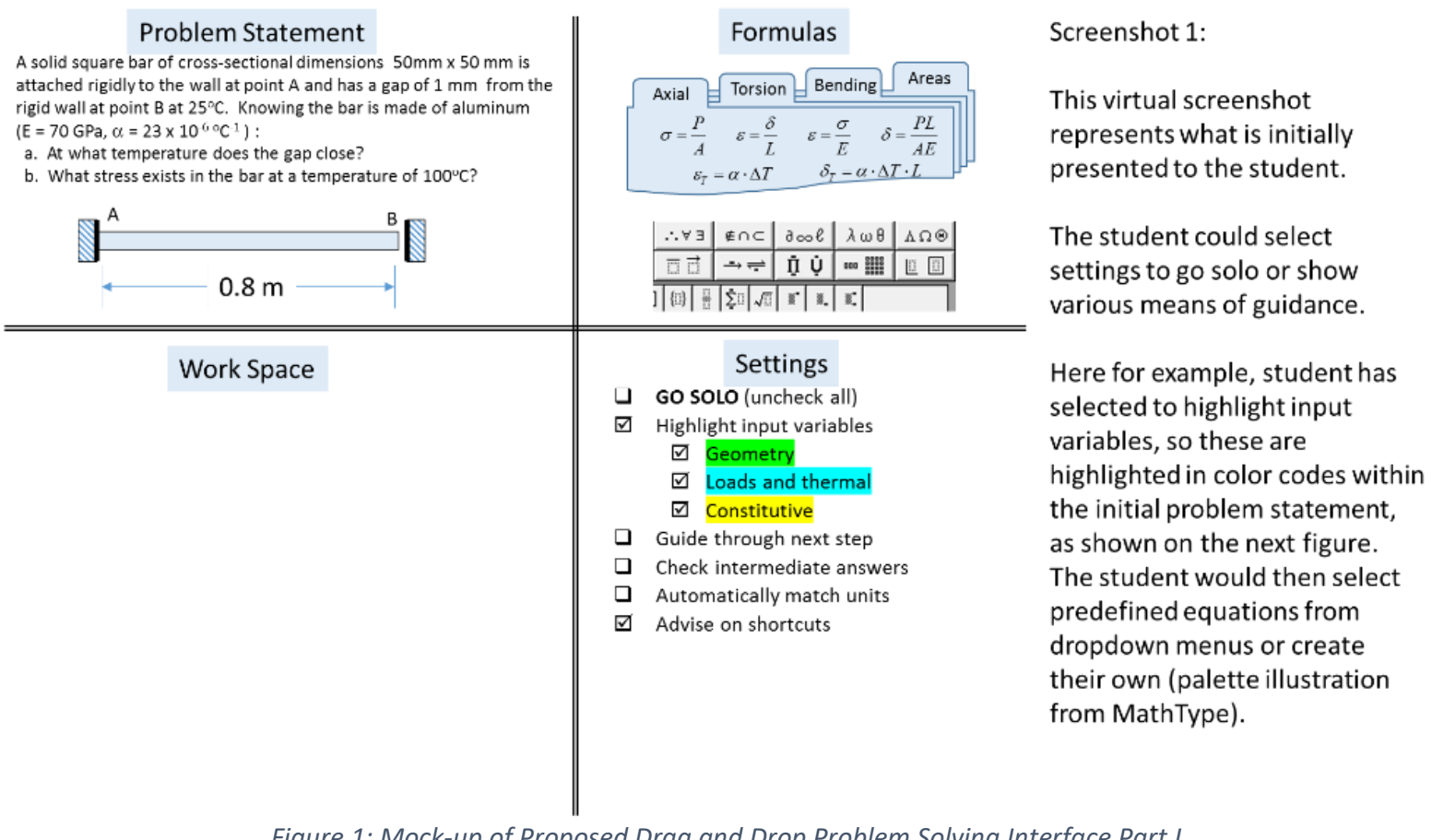

Figure 1: Mock-up of Proposed Drag and Drop Problem Solving Interface Part I 


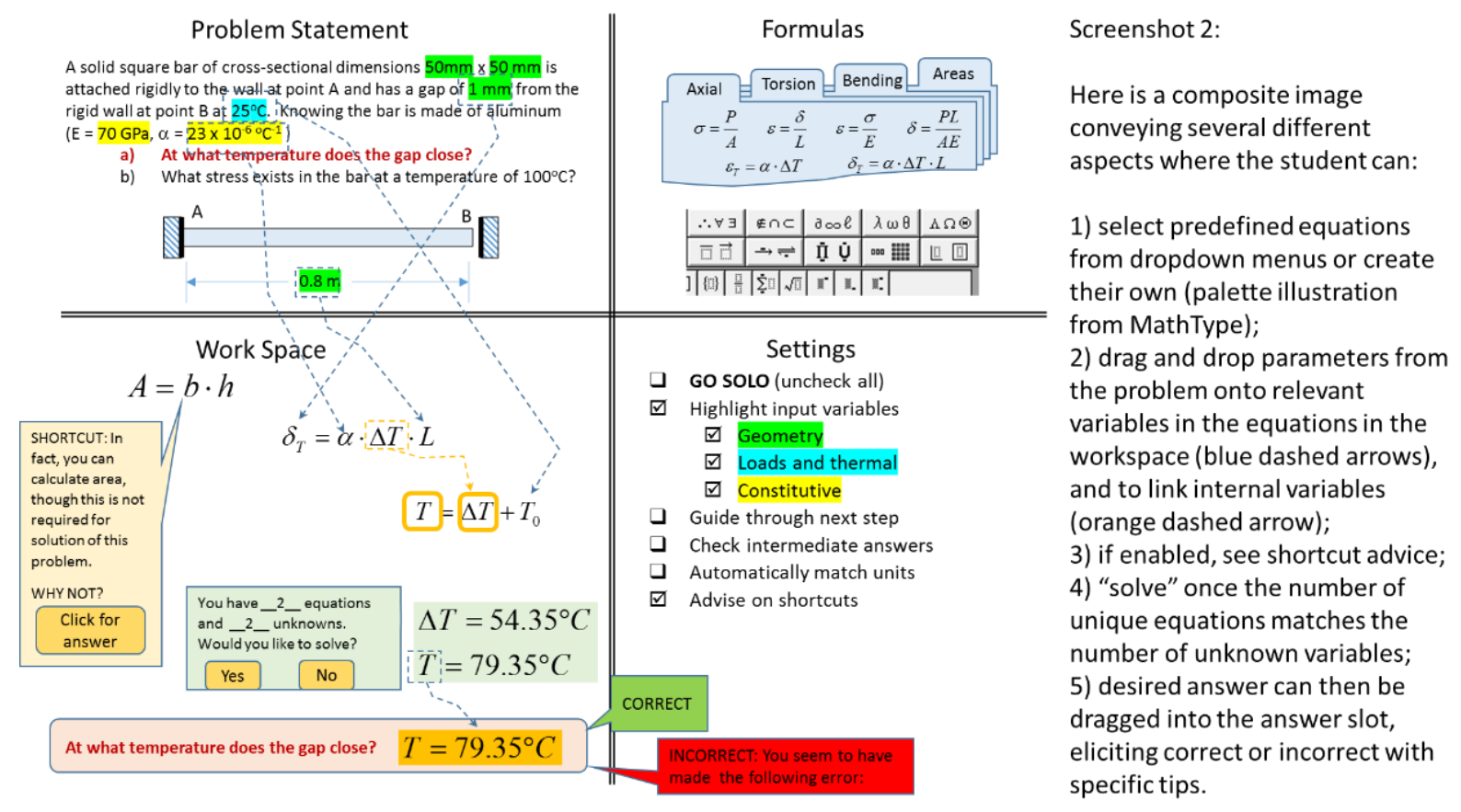

Figure 2: Mock-up of Proposed Drag and Drop Problem Solving Interface Part II

As we continue to work on our prototype, we are writing new items to reflect the concepts that our research has highlighted as problematic. This involves an item analysis of concepts covered on mid-terms and final exams that students tend to score the lowest on. One of the primary goals of this project is to use the innovation in order to systematically study how technology-rich environments can enhance the learning, teaching, and assessment of complex knowledge. Consequently, our exercises will be designed to enhance and accelerate conceptual learning (rather than use of rote algorithms) by minimizing the extraneous cognitive load of tedious calculations that can limit student ability to holistically understand how key concepts are interrelated.

\section{References:}

[1] National Academy of Engineering. 2004, The Engineer of 2020: Visions of Engineering in the New Century, National Academies Press, Washington, DC.

[2] Lattuca, L. R., Strauss, L. C., and Volkwein, J. F., 2006, "Getting in Sync: Faculty and Employer Perceptions from the National Study of EC2000," Int. J. Eng. Educ., 22(3), pp. 460-469.

[3] Lord, S. M., and Chen, J. C., 2014, "Curriculum Design in the Middle Years," Cambridge handbook of engineering education research, Cambridge University Press, New York, NY, pp. 181-195.

[4] Grohs, J., Soledad, M., Knight, D., and Case, S., 2016, "Understanding the Effects of Transferring In Statics Credit on Performance in Future Mechanics Courses," ASEE Conferences.

[5] Soledad, M., and Grohs, J., 2016, "Understanding Faculty Experiences in Teaching Large Classes: A Pilot Study on Faculty Perceptions of Teacher-Student Interaction in Foundational Engineering Courses," The 2nd Annual Teaching Large Classes Conference. 